\title{
BESCHREIBUNG UND PUBLIKATIONSPRINZIPIEN
}

\section{Das Buch Af 1}

Das Buch im Schmalfolio-Format $(41 \times 15,5 \mathrm{~cm})$ besteht aus 6 Heften zu je 24 Blättern, im ganzen also aus 144 Blättern. Das Papier ist stark und weiß; auf den Blättern 2-4, 7, 8, 12, 25, 27, 31-33, 35, 61, 67-71, 85-87, 90, 92, 93, 109, $110,112,113,115,133,136,139$ und 140 befindet sich als Wasserzeichen ein Jagdhorn. Der alte Einband fehlt. Die Hefte sind mit einem schwarz-grünen Faden an ein Stück rotbraunes Leder angenäht, das in der Form einer Aktentasche ausgeschnitten ist und ihnen als Hülle dient.

Auf dem Rücken des Buches ist ein Zettel angeklebt mit der Aufschrift: „Af N 1. Schuldbuch Veckinghusen 1399-1406“.

Die Blätter sind von Hildebrand Veckinchusen selbst sowie von neuerer Hand von 1 bis 144 durchnumeriert; beide Numerierungen fallen zusammen.

Hinter den Blättern 74, 87, 98, 108 und 117 sind Zettel eingelegt, welche ebenfalls alle von der Hand Veckinchusens beschrieben sind.

Die Handschrift ist unbeschädigt; nur vom Blatt 1 ist ein kleines Stück an der oberen Ecke abgerissen, der Text jedoch wurde hierdurch nicht berührt.

\section{Das Buch Af 6}

Das Buch im Schmalfolio-Format $(41 \times 15,5 \mathrm{~cm})$ besteht aus 10 Heften zu je 20 Blättern, somit im ganzen aus 200 Blättern.

Das Papier ist dem von Af 1 ähnlich; sein Wasserzeichen jedoch ein Einhornkopf. Es findet sich auf den Blättern 11, 13, 16, 17, 32, 34, 36, 38, 40, 51, 53, 55, $57,59,61,65,67-69,81,84,85,88,89,102,104,105,108,110,121,123,126$, $127,152-154,156,160-163,184$ und 188.

Der Einband ist aus weichem Pergament und hat die Form einer Aktentasche (mit einem Oberschlag). Eine Schnalle auf dem Vorderdeckel und ein Riemen auf dem Hinterdeckel sind heute abgerissen.

Die äußere Seite des Einbandes trägt von Veckinchusens Hand die Aufschrift:

„Int jar sevende jar op den ersten dach von october.

Int vorkopen"

Die Blätter dieses Buches wurden von Veckinchusen nicht numeriert; die Foliierung von 1 bis 200 stammt aus jüngster Zeit und wurde mit dem Bleistift vorgenommen. 
Auch hier sind Zettel eingelegt, und zwar hinter den Blättern 33, 44, 76, 80, 91, 128 und 140. Ihre Beschriftung stammt ebenfalls fast ausschließlich von Veckinchusen, nur der Zettel nach Blatt 44 trägt die Handschrift von Hans Wederman.

Die Beschädigungen sind ganz unbedeutend; der Unterteil des hinteren Einbanddeckels ist etwas zerdrückt. Weiter weisen die Blätter 189-200 im oberen Teil einige Spuren von Feuchtigkeit auf, und der untere Rand des letzten Heftes ist etwas lädiert; jedoch blieb der Text hiervon unberührt mit Ausnahme der untersten Zeile des letzten Blattes (Folio $200 \mathrm{v}$ ), von der nur einige unlesbare Buchstaben erhalten sind, der Rest ist abgerissen.

Die beiden Bücher Af 1 und Af 6 sind nur von einer Hand, nämlich der des Hildebrand Veckinchusen, geschrieben. Die Sprache ist mittelniederdeutsch, die Schrift eine lateinische Kursive aus dem Anfang des 15. Jh. Die Handschrift ist klein, stellenweise, wie etwa auf den ersten Seiten von Af 1, sehr klein, aber gut lesbar. Im ganzen ist sie in Af 6 etwas größer als in Af 1. Teils dünnerer, teils dickerer Konsistenz und von hellbrauner bis schwarzbrauner Farbe ist die Tinte. Die Federn sind unterschiedlich: die einen sehr spitz, die anderen etwas dicker, wovon die Handschrift etwas schmierig geworden ist. Die Tinten- und Federnuancen werden in den Noten angezeigt, besonders dann, wenn sie zum Verständnis des Inhalts und der Struktur des Buches, z. B. bei der Scheidung von Grundtext und späteren Zutaten, behilflich sein können.

Veckinchusen ist mit dem Papier nicht sehr sparsam umgegangen: Eine Anzahl von Seiten besonders in Af 6 ist leer geblieben, und viele sind bei weitem nicht ganz ausgefüllt. Manchmal befinden sich nur einige Zeilen auf einer Seite.

Eintragungen ohne Beziehung zum Inhalt des ursprünglichen Textes eine in anderen uns bekannten Handelsbüchern der Zeit übliche Ausnützung des freien Raumes - gibt es nur sehr selten.

Die zahlreichen freigebliebenen Seiten in Af 6 sind durch die Tendenz zu erklären, das Buch aus einem Memorandum in ein Konto-Buch zu verwandeln und deshalb die Seiten im voraus für die verschiedenen Gruppen von Eintragungen aufzuteilen.

Bei der Vorbereitung des Textes für den Druck wurde zunächst darauf geachtet, die Beschaffenheit des Originals möglichst getreu wiederzugeben, nämlich die Anordnung der Seiten, innerhalb der Seiten die Anordnung der Zeilen, die Abstände zwischen diesen und zwischen den Posten und endlich innerhalb der Zeilen die Abstände zwischen den einzelnen Gruppen der Wörter oder Zeichen.

Aus technischen Gründen mußte man aber darauf verzichten, den Text so zu gestalten wie in den letzten Prachtausgaben der italienischen Handelsbücher, wo jede Seite des Buches mit der entsprechenden Seite des Originals identisch ist. Die Struktur des Handelsbuches wäre bei einem solchen Verfahren viel besser zur Anschauung gekommen; andererseits aber - und das gab den Ausschlag - 
blieben dann wegen der sehr ungleichen Beschriftung der einzelnen Blätter des Originals, welche manchmal nur fünf Zeilen beträgt, einige Seiten unseres Buches nahezu unbedruckt und die meisten bis auf ein Drittel leer, was seinen Umfang noch einmal mindestens um die Hälfte hätte anwachsen lassen. Aus denselben Gründen konnte der Abstand zwischen den Posten, der im Original öfter mehrere Zentimeter breit ist, nur durch einen Absatz und an einigen Stellen durch eine Anmerkung angezeigt werden. Die Anordnung der Zeilen ist jedoch streng eingehalten; jede Zeile des Druckes entspricht genau der entsprechenden des Originals. Sie sind im Druck numeriert worden, und zwar einzeln für jede Seite des Originals. Da in den Namen-, Sach- und Ortsregistern bei jedem Stichwort Folium und Zeile ebenfalls genau angezeigt sind, wird, wie ich hoffe, das Auffinden dieser Stichworte im Text wesentlich erleichtert.

Die Zwischenräume innerhalb der Zeilen sind besonders bei Zahlen (Preisen, Gesamtbeträgen usw.) kenntlich gemacht - haben wir es hier doch schon mit einer gut ausgearbeiteten Form der Buchung zu tun.

Bei der Textgestaltung habe ich mich im großen und ganzen an die üblichen Editionsregeln gehalten, wie sie bei den großen hansischen Quellenveröffentlichungen angewendet werden. Auf einige Schwierigkeiten, welche der Text bietet, obwohl er in gut lesbarer Kursive geschrieben ist, muß allerdings noch aufmerksam gemacht werden.

Die Majuskeln „B“ und „V“ sind am Anfang völlig gleich geschrieben, die Lesung einiger Eigennamen bleibt daher unsicher: man kann so „Brunsberch“ oder „Vrunsberch“, „Bull“ oder „Vull“ lesen.

Auch die Kleinbuchstaben „c" und „t" sind nicht zu unterscheiden, wasin einigen Fällen erhebliche Schwierigkeiten bereitet. So muß etwa offenbleiben, ob der Name eines Geschäftspartners von Veckinchusen als „Scalin“, „Stalin“ oder, wenn der Punkt über dem i fehlt, vielleicht auch als „Scalm“ oder „Stalm“ zu lesen ist.

Zwei senkrechte Striche stehen für „n“ und „, Zeichen steht, auch für „u“. So kann man leicht dazu verführt werden, mit W. Stieda „Stoven“ statt „Sconen“ oder mit dem belgischen Archivar Gilliodts van Severen „,venerwamme“ statt „beverwamme“ zu lesen.

Wenn, was nicht selten vorkommt, in der Kombination von drei Strichen der Punkt über dem i fehlt oder nicht richtig gestellt ist, sind „ni“, „in“ und „m“ nicht voneinander zu unterscheiden. Dort können der Dativ Singular des Personalpronomens „em" (ihm) und der Dativ Plural „ein“ (ihnen) leicht verwechselt werden. Ich habe daher, um Mißverständnissen vorzubeugen, die Schreibung dem Sinne nach unifiziert.

Für die zweifelhaften Eigennamen wurden, wie es in solchen Fällen üblich ist, in weiteren Quellen Angaben gesucht, welche die eine oder andere Lösung sichern. Blieb dies erfolglos, so wurde, wenn möglich, eine Variante für den ganzen Text angenommen. Sie steht dann auch im Namenregister an erster Stelle, die übrigen eventuell auftretenden sind in einer Klammer dahinter ein-

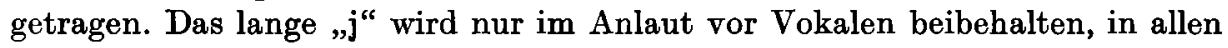
anderen Fällen durch ein kurzes , ,i“ ersetzt. „U“ und „, ${ }^{“}$ werden, des Originals 
ungeachtet, nach der Aussprache gebraucht; es wird also nicht „vnde“, sondern „unde“, nicht „vns", sondern „uns" gedruckt.

Alle Abkürzungen wurden aufgelöst, nur jene für die Geld- und Münzbezeichnungen („M. lub.“, „M. sund.“ u. a.) unverändert wiedergegeben.

Die sehr seltenen, zur Klärung des Originaltextes nötigen Zusätze des Editors stehen in eckigen Klammern.

Während sein Nürnberger Zeitgenosse Hilpot Kress bereits 1392 die arabischen Ziffern verwendete, bediente sich Veckinchusen noch der römischen Zahlen. Sie werden im folgenden durch arabische ersetzt, da diese die Lesbarkeit und das Verständnis bedeutend erleichtern. 


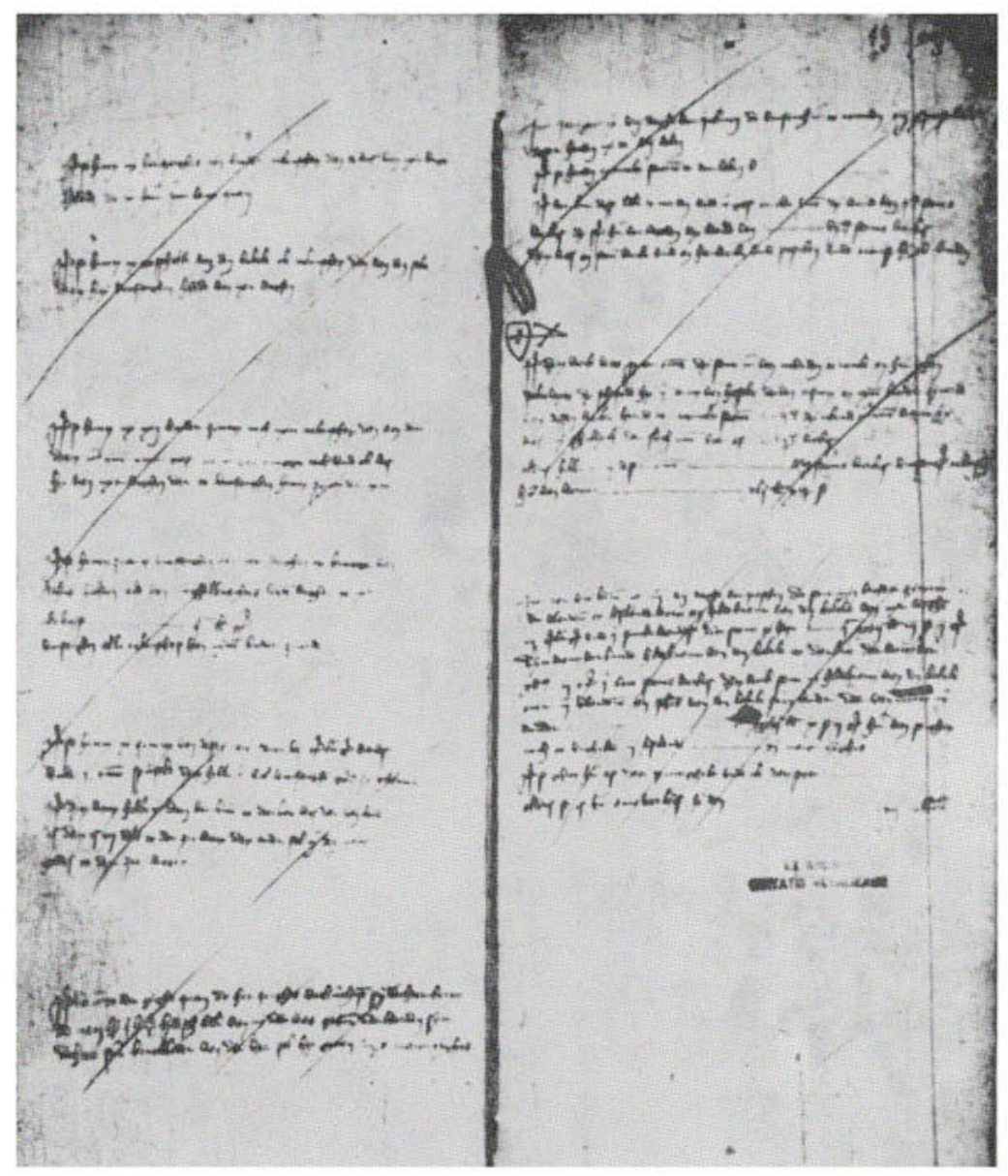

Aus Af 1 Folio 13 


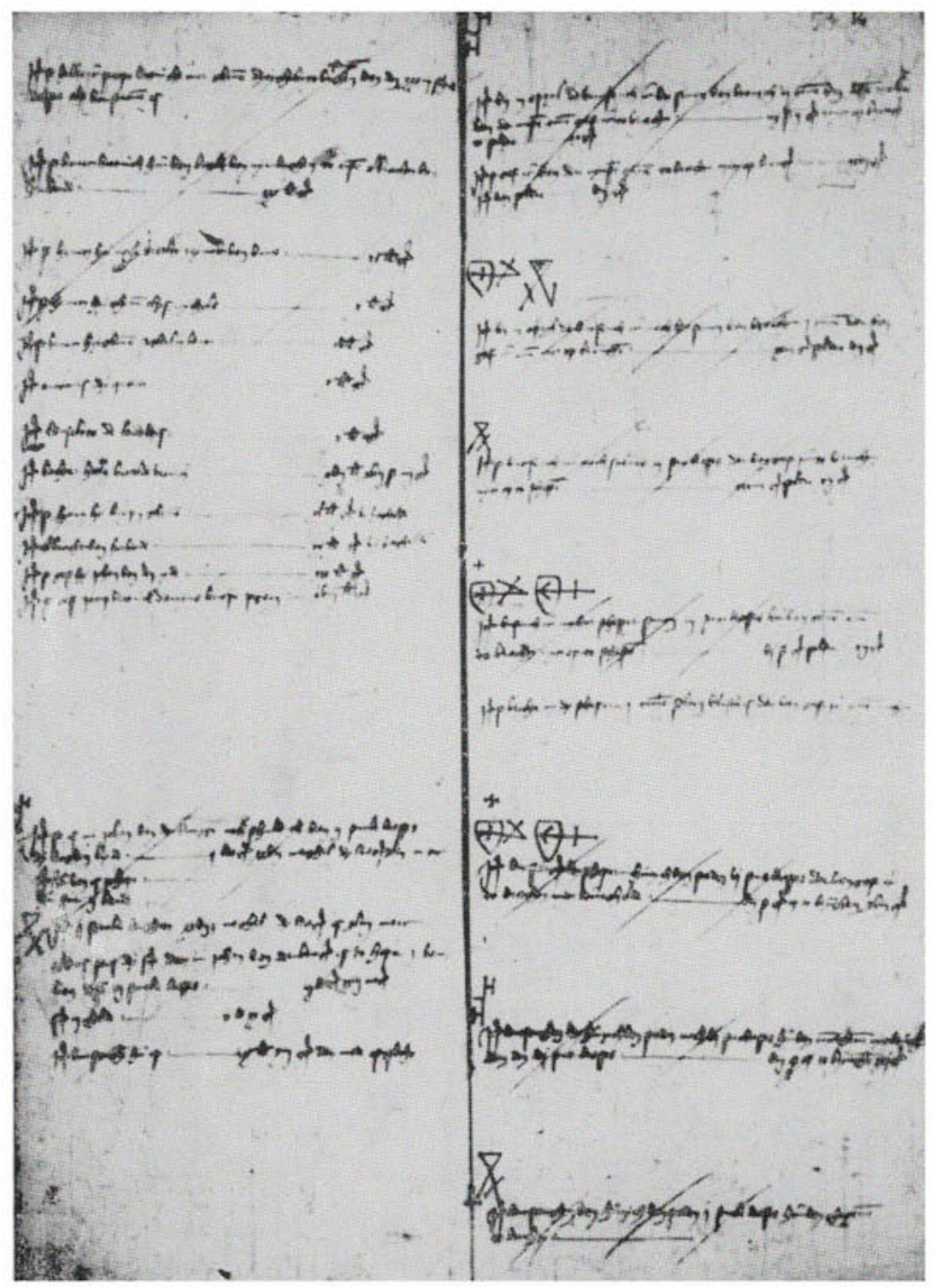

Aus Af 1 Folio 59 


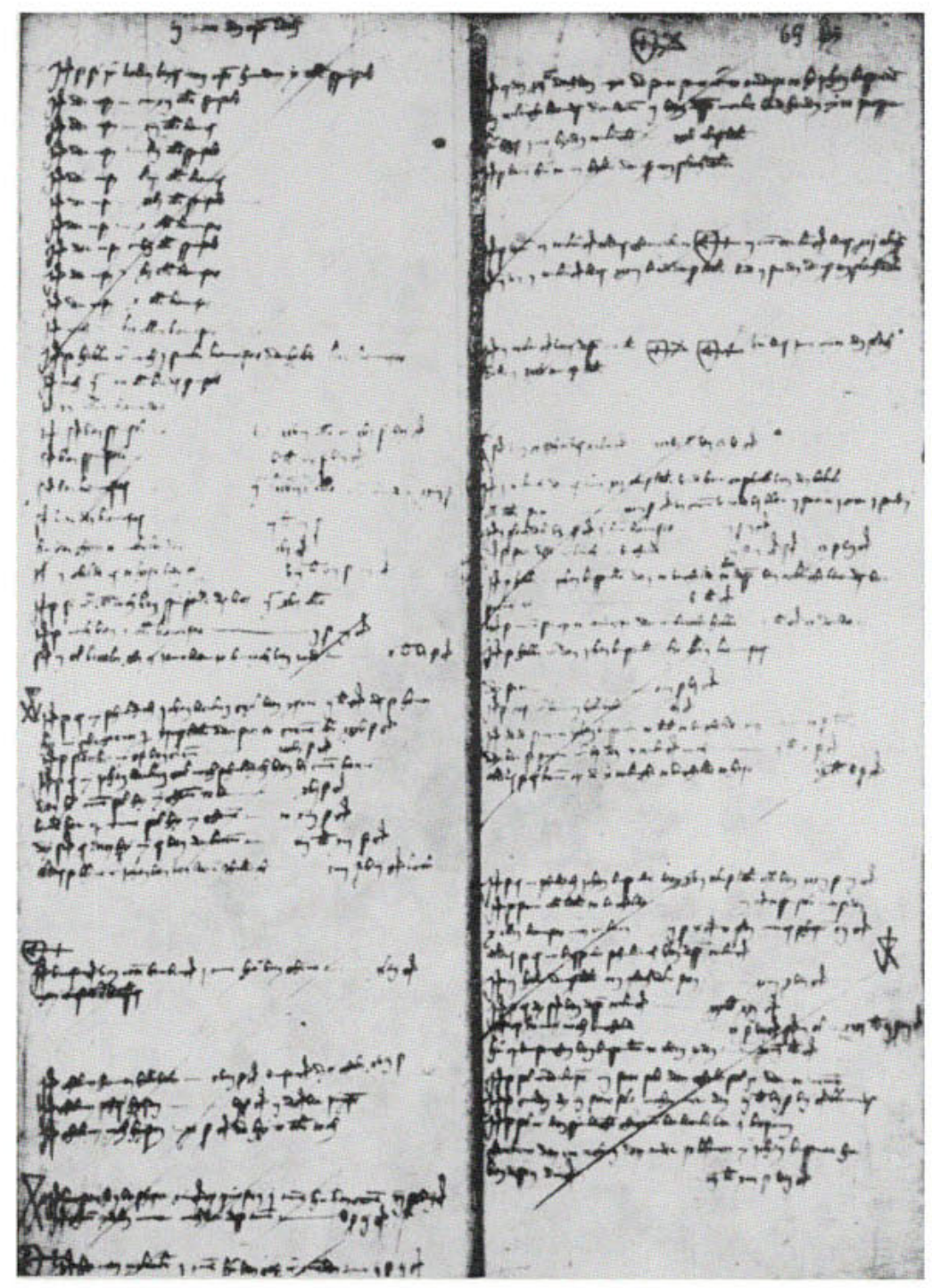

Aus Af 1 Folio 69 


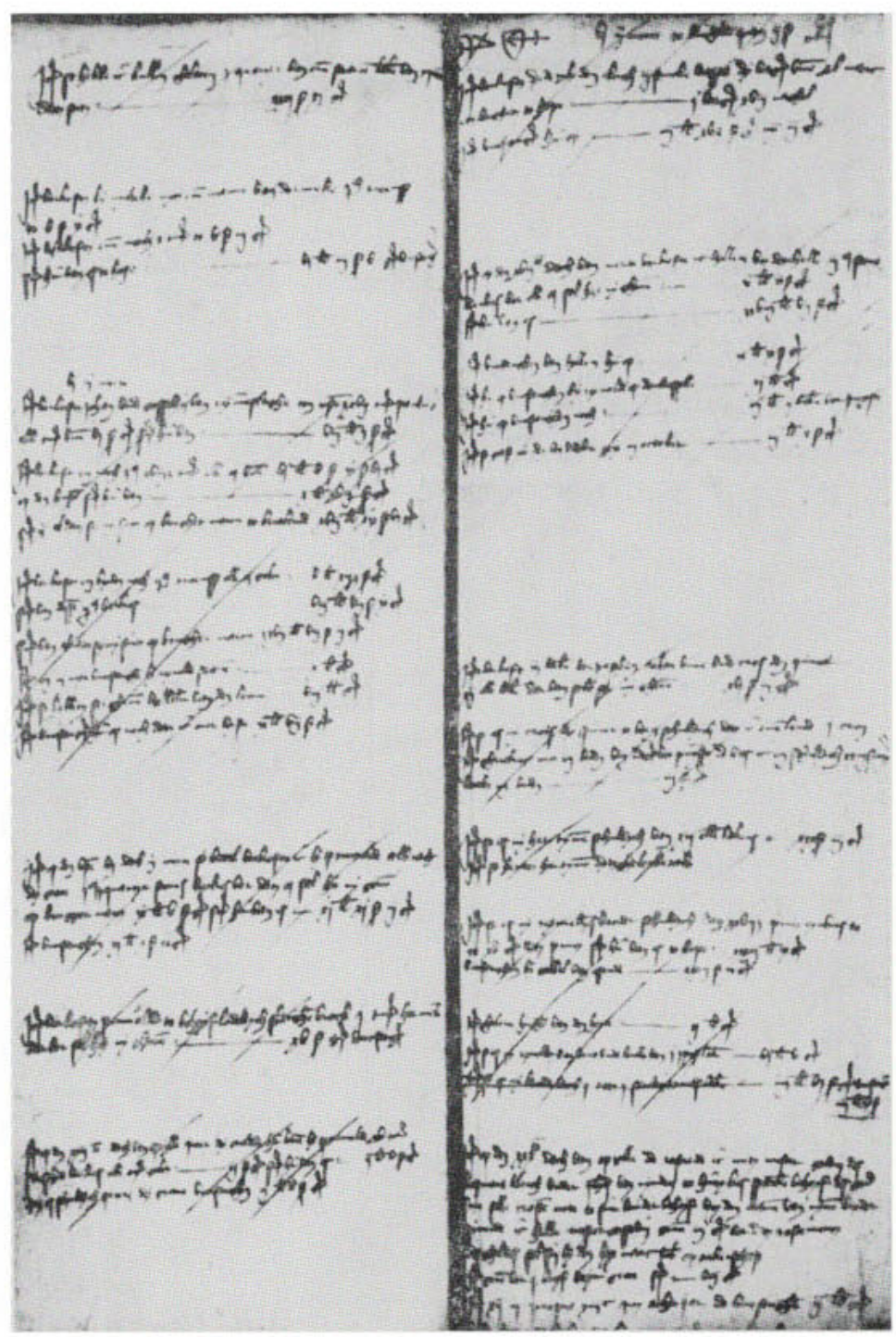

Aus Af 1 Folio 91 